Voix et Images

voixetimages

\title{
BIBLIOGRAPHIE DE PATRICE DESBIENS
}

\section{Mathieu Simard et Jean Marc Larivière}

Volume 44, numéro 3 (132), printemps-été 2019

Patrice Desbiens

URI : https://id.erudit.org/iderudit/1064626ar

DOI : https://doi.org/10.7202/1064626ar

Aller au sommaire du numéro

Éditeur(s)

Université du Québec à Montréal

ISSN

0318-9201 (imprimé)

1705-933X (numérique)

Découvrir la revue

Citer ce document

Simard, M. \& Larivière, J. M. (2019). BIBLIOGRAPHIE DE PATRICE DESBIENS.

Voix et Images, 44(3), 113-133. https://doi.org/10.7202/1064626ar d'utilisation que vous pouvez consulter en ligne.

https://apropos.erudit.org/fr/usagers/politique-dutilisation/ 


\title{
B I B L I OGR A PH IE DE PATRICE DESBIENS
}

\author{
$+++$ \\ MATHIEU SIMARD \\ Université d'Ottawa \\ avec la collaboration de \\ JEAN MARC LARIVIËRE
}

\section{E U V RES ${ }^{1}$}

\section{A. Livres}

+ Cimetière de l'œil, Saint-Marc-des-Carrières, édition à compte d'auteur, 1972, [s. p.].

+ Larmes de rasoir, Québec, édition à compte d'auteur, 1973, [s. p.].

+ Ici, Québec, Éditions à Mitaine [à compte d'auteur], 1974, 30 p.

+ Les conséquences de la vie, Sudbury, Prise de parole, 1977, 47 p.

+ L'espace qui reste, Sudbury, Prise de parole, 1979, 92 p.

+ L'homme invisible/The Invisible Man, édition bilingue, Sudbury/Moonbeam, Prise de parole/ Penumbra Press, 1981, 46 p. Rééditions chez Prise de parole en 1997, 2001 et 2008 (collection «BCF. Bibliothèque canadienne-française»; l'édition de 2008 est accompagnée d'une préface de Johanne Melançon), sous le titre L'homme invisible/The Invisible Man, suivi de Les cascadeurs de l'amour.

+ Sudbury: textes 1981-1983, Sudbury, Prise de parole, 1983, 63 p.

+ Dans l'après-midi cardiaque, Sudbury, Prise de parole, 1985, 77 p.

+ Les cascadeurs de l'amour, Sudbury, Prise de parole, coll. «De-Ville», 1987, 73 p.

+ Poèmes anglais, Sudbury, Prise de parole, coll. «De-Ville», 1988, 62 p.

+ Amour ambulance, Trois-Rivières, Écrits des Forges, 1989, 85 p. Traduit en espagnol par Gabriel Martin, Amor ambulancia/Amour ambulance, Tlaquepaque/Trois-Rivières, Mantis Editores/ Écrits des Forges, coll. «Terredades», 2010, 103 p.

+ Un pépin de pomme sur un poêle à bois, précédé de Grosse guitare rouge, précédé de Le pays de personne, Sudbury, Prise de parole, 1995, 205 p.

+ L'effet de la pluie poussée par le vent sur les bâtiments, sérigraphie originale d'André Éliceiry, Québec, Docteur Sax [à compte d'auteur], 1997, 24 p. Réédition: L'effet de la pluie poussée par le vent sur les bâtiments, Outremont, Lanctôt, 1999, 129 p.

+ La fissure de la fiction, Sudbury, Prise de parole, 1997, 49 p.

+ Rouleaux de printemps, Sudbury, Prise de parole, 1999, 95 p. Réédition en 2015.

1 Je remercie chaleureusement Jean Marc Larivière, qui a répertorié la plupart des textes de Patrice Desbiens en périodiques et collectifs présentés dans les sections I-B et I-C de la présente bibliographie. Certains des périodiques de cette section étant difficiles d'accès (Steak haché, Harbinger, Réaction, etc.), le numéro de la page où se trouve le poème recensé est parfois absent. Il en va de même pour quelques références (beaucoup plus rares cependant) dans d'autres sections de la présente bibliographie. 
+ Sudbury (poèmes 1979-1985), Sudbury, Prise de parole, 2000, 259 p. (Ouvrage composé de L'espace qui reste [1979]; Sudbury: textes 1981-1983 [1983]; et Dans l'après-midi cardiaque [1985]). Rééditions en 2007 et 2013.

+ Bleu comme un feu, Sudbury, Prise de parole, 2001, 67 p.

+ Hennissements, Sudbury, Prise de parole, 2002, 103 p.

+ La troisième Langue de feu, avec des illustrations de Robert Daigneault, Montréal, Steak haché, 2003, 63 p. (Livre publié à l'occasion du prix Langue de feu. Il s'agit d'une sélection de textes de Patrice Desbiens parus dans la revue Steak haché entre 1998 et 2003).

+ Désâmé, Sudbury, Prise de parole, 2005, 60 p.

+ Déchu de rien, Montréal, édition à compte d'auteur, 2006, 25 p.

+ Inédits de vidé, Montréal, édition à compte d'auteur, 2006, 10 p.

+ Leçons de noyade, Montréal, édition à compte d'auteur, 2006, 23 p.

+ En temps et lieux, Montréal, L'Oie de Cravan, 2007, 57 p.

+ [dekala3]/Décalage, Sudbury, Prise de parole, 2008, [65] p.

+ En temps et lieux 2, Montréal, L'Oie de Cravan, 2008, 55 p.

+ En temps et lieux 3. Le dernier cahier, Montréal, L'Oie de Cravan, 2009, 57 p.

+ Poèmes anglais, suivi de Le pays de personne, suivi de La fissure de la fiction, Sudbury, Prise de parole, coll. «BCF. Bibliothèque canadienne-française», 2010, 223 p.

+ Pour de vrai, Montréal, L'Oie de Cravan, 2011, 60 p.

+ Un pépin de pomme sur un poêle à bois, Sudbury, Prise de parole, 2011, 59 p.

+ Tout ça m'assassine. Courtes pièces sur l'air du temps, sous la direction de Renaud Plante, avec «Poèmes, première partie» et «Poèmes, deuxième partie» de Patrice Desbiens, «La déroute» de Dominic Champagne et «Confession d'un cassé» de Pierre Lefebvre, Montréal, Somme toute, coll. «Répliques», 2013, 133 p.

+ Les abats du jour, Montréal, L'Oie de Cravan, 2013, 71 p.

+ Vallée des cicatrices, Montréal, L'Oie de Cravan, 2015, 57 p.

+ Le quotidien du poète, Sudbury, Prise de parole, 2016, 56 p.

+ En temps et lieux. Les cahiers complets, Montréal, L'Oie de Cravan, coll. «Petites pattes à ponts», 2017, $135 \mathrm{p}$.

+ Sous un ciel couleur cayenne, Sudbury, Prise de parole, coll. «Poésie», 2017, 58 p.

+ The Art of Disappearing, Victoria, Ekstasis Editions, 2017, 102 p.

\section{B. Recueils en format audio ou hybride}

+ La cuisine de la poésie présente: Patrice Desbiens, Sudbury, Prise de parole, 1985, audiocassette.

+ Patrice Desbiens et les Moyens du bord, poèmes de l'auteur issus de différents recueils écrits entre 1974 et 1995 sur des musiques improvisées par Jean Derome, Guillaume Dostaler, René Lussier et Pierre Tanguay, Montréal, DAME, 1999, 1 disque numérique.

+ Grosse guitare rouge, livre et disque, musique de René Lussier, Sudbury, Prise de parole, 2004. 


\section{Poèmes parus dans des périodiques et des collectif s ${ }^{2}$}

+ «Beautiful Burning Angels...» et «The People Walk Within...», Harbinger, juillet-août 1968 [signés Patrick Desbiens].

+ «My Bathrobe Whispers to Me...» et «The Purpose of the Poem», Harbinger, septembre 1968 [signés Patrick Desbiens].

+ «Poems by Patrice Desbiens: Nightbeat Nighttime...; It's a Grey Dog Day...; Someone's Killed the Sun...; The Dull Song of a Falling Bird...», Harbinger, hors série, 1968.

+ "Sun Comes Up...» et «It Is 1:21 in the Morning...», The Eye Opener, vol. III, no 15, 1969, p. 7.

+ "A Four-Story House...», The Eye Opener, vol. III, n 16, 1969, p. 8 [signé Pat Desbiens].

+ «Bring our Children Down...», «Aube», «Un vaste rire...» et «Mes pieds fatigues», The Eye Opener, vol. III, nº 17, 1969, p. 13-14.

+ «Many Time Many Time...», The Eye Opener, vol. III, n 18, 1969, p. 10.

+ «Oh, Cinema Queen...», The Eye Opener, vol. III, n 20, 1969, p. 5.

+ «Collage (Improvised)», The Eye Opener, vol. III, n 21, 1969, p. 7.

+ "Boarded Up into Evenings», avec deux poèmes de Bruce Cockburn, The Eye Opener, vol. III, n 24,1969 , p. 9.

+ «Flesh Constructions», The Eye Opener, vol. III, n 35, 1970, p. 8-9.

+ «Chantez, dansez...», The Eye Opener, vol. IV, n 8, 1970, p. 10.

+ "Part of a Play», The Eye Opener, vol. IV, n 10, 1971, p. 6-7.

+ «My Girl She's a... » et «I Laugh and the Haha's... », The Eye Opener, vol. V, n 6, 1971, p. 5.

+ «Rouge», Ébauches, ACFO, n 10, 1977.

+ «Tonto in Toronto » et "Autumn from the Kitchen Window», 2 O'clock Rap, vol. V, Toronto Public Library, 1977. [Réédition de «Tonto in Toronto» dans Ward 7 News, vol. IX, n 10, 1978.]

+ «Everything» et «Red», Ward 7 News, vol. VIII, n 2, 1977.

+ «It's Very Pretty» et "A Short Mystery Story», Ward 7 News, vol. VIII, n 10, 1977.

+ «We Are Two Stains...» et «No Way of the...», Poetry Toronto Newsletter, n 24, 1977.

+ «Les périls de Pauline», "Notes de l'odeur», "Au fond (des choses)», «Moto Québec», «Ketchup», «Et le Dieu bleu», «Mariage» et «Mata Hari Kiri», Black Moss, vol. II, n 4, Mooskek Reader, 1978.

+ «Le rêve de Noël d’un Franco-Ontarien», L’Express de Toronto, vol. III, n 51, 1978.

+ "L'hiver nous fait comme...», L’Express de Toronto, vol. IV, n 2, 1979.

+ «Aller-retour», L'Express, 28 septembre au 4 octobre 1979, p. 13.

+ "Quand j'étais à Timmins», "Ce que l'hiver nous fait», «Souvent quand je pense à elle», "Chapitre perdu d'un roman perdu », «L'humidité du désespoir», "Audie Murphy», «Ici et là», «Dans un restaurant de Toronto», «Danielle», «Parti pour la gloire», «Dimanche à Cornwall», «Déjeuner», «La pluie et Marguerite» et «On», La Souche, 1981. (Recueil publié avec Neil Lefaivre, Daniel Rhéaume, Michel Dallaire et Maurice Lapointe, alors étudiants à l'Université Laurentienne, grâce à une subvention du Secrétariat d'État.)

+ «Biscuit soda", «Des ruisseaux», «Entre deux bières», "Des églises en chaleur», «Pour toi pourquoi Nicole», «Downtown», «Le matin 3-étoiles de Toronto», «Testicules de Texaco ou Avco Finance will fuck», «Le brave apache», «Avec un seul regard, elle», «Une poitrine en

2 La toute première publication de Patrice Desbiens serait parue dans Toronto Telegram - non dans Harbinger, comme pourrait le laisser entendre cette section de la bibliographie. Ce premier poème demeure toutefois introuvable à ce jour. 
feu», «Le meilleur des mondes», "Claude, Maurice, Simon, Moi, la rue St-Jean, Québec, etc.» et «On a grimpé dans l'arbre», Anus, vol. I, nº 6, juin-juillet 1981.

+ «Je me souviens...» et «Dans la Coulson un lundi soir», Réactions, vol. IX, n 3, 1981.

+ «Comme un refrain», "Un autre matin se cogne les orteils» et «Poitrine de feu», Réactions, vol. X, n 4, 1982.

+ "Les os de mes ancêtres", «Les conséquences de la vie», "Ma mère dansait le Charleston", «Mariage», «La machine à Coke», «Le rêve de la plupart», «L'espace qui reste», «L'homme invisible/The Invisible Man", Poèmes et chansons du Nouvel-Ontario, Sudbury, Prise de parole, 1983, p. 34-44.

+ «Je regarde par la fenêtre...», «La subtilité...», «Les rues sont en feu...», «Le lit défait...» et « J'ai le rock and roll», Anus, vol. III, n 1, 1983.

+ «Portrait», Liaison, n 29, hiver 1983-1984, p. 23.

+ «Poèmes anglais», Rauque, $\mathrm{n}^{\circ} 1,1984$.

+ «Attentat» et «Une tranche de pain», Marie-José Trudel et Évelyne Tran (dir.), De la neige au soleil. Un recueil de quatre-vingt-six poèmes du Québec et de la francophonie des Amériques, avec un poème préface de Gilles Vigneault et des illustrations de Christine Dzieczyk, Montréal/ Paris, Ville-Marie/Nathan, coll. «Publications PPMF Laval», 1984, [s. p.].

+ «La chérie canadienne», Rachelle Renaud (dir.), Tout près d'ici, Sudbury, Prise de parole, 1984.

+ «Hier, Vendredi saint et tout», N'importe quelle route, vol. I, n 2, 1987, p. 30-31.

+ «Histoire d'Aimée», Rauque, n 7, 1987.

+ «Déjeuner», Liaison, n 47, juin 1988, p. 39.

+ «Cambrian Country», «Le bruit de l'Amérique» et «Jaco», N'importe quelle route, vol. II, n², 1988, p. 38-39.

+ «Incident en Acadie», N'importe quelle route, vol. III, n² 2, 1989, p. 33.

+ «Hier, Vendredi saint et tout» et «Ā CBON...», Québec Kérouac Blues, Trois-Rivières, Écrits des Forges, 1989, p. 36-44.

+ «Les os de mes ancêtres», «L'aéroport de Sudbury», «Hearst», «Nicole» et «Devinette», Anthony Mollica et Bernadette LaRochelle (dir.), Reflets d'un pays. Poèmes et chansons, préface de Cécile Cloutier, Welland/Lewiston, Éditions Soleil, 1990, p. 55, 96, 98, 172 et 187.

+ «La voiture frappe le mur...», «Le bruit que fait...», «Une cigarette est allumée...», «Acte de bravoure... ", «Une promenade avec aimée», "Aimée et le prince charmant» et "Aimée et la pleine lune», Louise Blouin (dir.), Des mots pour rêver. Anthologie de poésie québécoise, Trois-Rivières/Montréal, Écrits des Forges/Éditions Pierre Tisseyre, coll. «Conquêtes», 1990, p. 55-63.

+ «Pourrir sans mourir» [avec Michel X Côté], Richard Desjardins (dir.), Bande sonore du film Le Party de Pierre Falardeau, Montréal, Justin Time, 1990, disque compact.

+ «Les os de mes ancêtres», «Mariage», «La chérie canadienne», «Le vrai drame de la chaleur», «Les tambours sont muets... », «Treize heures d'autobus... », "Timmins» et «Hôtel Hystérie 3 », René Dionne (dir.), Anthologie de la poésie franco-ontarienne des origines à nos jours, Sudbury, Prise de parole, 1991, p. 110-118.

+ «Tu rentres dans un œil...», «Nous sommes condamnés...», «Tu passes du regard...», «Mon corps est une prison... » et "La somme de tout», Cahier Folie/Culture, $n^{\circ} 2$, textes et productions visuelles dans un coffret de métal et de feutre, 1991.

+ «La somme de tout» [1. «L'angle obtus»; 2. «L'angle droit»; et 3. «L'hypoténuse»], Liaison, $\mathrm{n}^{\circ} 65$, janvier 1992, p. 23. 
+ «Je/Je suis/...», Cahiers Folie/Culture, n 3, textes et productions visuelles dans un rouleau de sous-tapis, 1992.

+ «tu es fragile comme une statue...», Estuaire, n ${ }^{68}$, printemps 1993, p. 19-25.

+ "Cambrian Country», "Howard Johnson Poems» et "Eux, le barman et le poète», LittéRéalité, vol. VII, nos $1-2,1995$, p. 120-122.

+ «Tu m'enlèves... », Liaison, n 82, mai 1995, p. 33.

+ «Hôtel chancelant», «Éducation permanente», «Petit bouguie bleu», «Sel vinaigre drapeau», "Avec un seul regard, elle», "Papa a raison avec Black \& Decker», «Les femmes dans ma vie jusqu'ici», «La vie sourit comme un taxi», «Josée Yvon est... », Exit, nº 1, 1995.

+ «La mort de Colette» et «Jésus de Sudbury », Mœbius, n 67, printemps 1996, p. 77-79 et p. 80-81.

+ "Des nouvelles de Sudbury», "L'effet de la pluie poussée par le vent sur les bâtiments", Estuaire, n 85 , janvier 1997, p. 18-24.

+ «À Gilbert Langevin. Poévivant», Le Sabord, n 47, 1997, p. 12.

+ «Rhum et Coke», «Tomahawk», «La folie comme raccourci» et «La cigale joue et gagne», Liaison, n 95, janvier 1998.

+ «Les chiens et la poésie», Steak haché, n 5, 1998.

+ «La facilité des lèvres», Steak haché, nº 6, 1998.

+ «Pleine lune sur St-Denis» et "Ceci et cela», Steak haché, n 7, 1998.

+ «Une seconde dans la vie d'un poème», «Si tu étais» et "Poisson d'avril», Steak haché, n 8, 1998.

+ «Hennissements», Steak haché, nº 9, 1999.

+ «Baltimore, Babe, Baltimore», Steak haché, n 10, 1999.

+ "L'électricité», «Astronomie», «Poésie (1)» et «Quelque chose dans l'air», Steak haché, no 11, 1999.

+ «Corde raide», Steak haché, nº 12, 1999.

+ «Nuit», Steak haché, no 13, 1999.

+ «Ils ne savent pas», "Pays à poil» et «Si tout d'un coup», Steak haché, n 14, 1999.

+ «Déjeuner», «La paix au Moyen-Orient», «L'humidité du désespoir», «Les canards de l'insouciance» et «Ce que l'hiver nous fait», Steak haché, n 15, 1999.

+ «Maya», «Insérez titre ici», «Poésie» et "Chandelle», Steak haché, n 16, 1999.

+ «Wouwou», «Douceronne» et «Une fois pour tout», Steak haché, n 17, 1999.

+ «La balade du veau d'or et de la vache qui rit», Steak haché, no 18, 1999.

+ "Bleu comme un feu», Steak haché, n 19, 1999.

+ «Mon chat», Steak haché, nº 20, 1999.

+ «Miles à Montréal, moi à Sudbury», Steak haché, n 22, 2000.

+ «T'es pas ma blonde», Steak haché, n 23, 2000.

+ «Brise par brise», Steak haché, n² 24, 2000.

+ «Écureuil folle», Steak haché, n 25, 2000.

+ «Pas de deux sans toi» et «Tu fais», Steak haché, n 26, 2000.

+ «Premier juillet», «Homme» et «Giblotte», Steak haché, n 27, 2000.

+ «Tomahawk», «Maquille la montagne» et «Sur une plage», Steak haché, n 28, 2000.

+ «Sujet verbe» et «Ce courant de ton regard», Steak haché, n² 29, 2000.

+ «Bonsoir, bonne nuit» et «Un chat», Steak haché, n 30, 2000.

+ «Nuit blanche», "Ce que l'hiver nous fait», «Les petits ganglions», «Les sauterelles du souvenir», Steak haché, n 31, 2000. 
+ «Ā vol d'oiseau», «Même toi », «Millenium», «Lundi-Monday » et «Correspondance», Steak haché, n 32, 2000.

+ "L'électricité», "Quelque chose dans l'air», "Corde raide», «Nuit», «Ils ne savent pas», «La paix au Moyen-Orient», "Miles à Montréal, moi à Sudbury » et "Brise par brise», La vérité se passe un doigt. Steak haché anthologique, Notre-Dame-des-Neiges, Éditions Trois-Pistoles, coll. «Inédits», 2000, p. 70, p. 78, p. 95-96, p. 106, p. 110-111, p. 119, p. 189-190, p. 208-209.

+ «Dedans le ciel», «Le sourire de tes fesses» et «Hennissements», Steak haché, no 33, 2001.

+ «Fabienne Thibault au centre d'achats», «Sleeping», «Toupie qui dort» et «Poisson messager», Steak haché, nº 34, 2001.

+ «Portage», «J'ai le goût d’une cabane» et «Fais que...», Steak haché, nº 35, 2001.

+ «Face-off», "Rien d'trop beau», "Gibelotte» et «Wouwou», Mœbius, n 89, printemps 2001, p. 33-36.

+ «Panne», «Onze rue des Feuillantines», «Poser», «Filet de soul», Steak haché, n 36, 2001.

+ «Face à face» et «Rapido», Steak haché, n 37, 2001.

+ «Un extrait du grand livre de la schizophrénie appliquée», «Derrières les heures», «Claire, comme la lune» et «Le le...», Steak haché, n 38, 2001.

+ «Rencontre» et «Rien d'autre à faire», Steak haché, n 39, 2001.

+ «Cours de chant», «Dans le silence» et «Sujet verbe», Steak haché, n 40, 2001.

+ "Dieu à Timmins», «Kiwi» et «True Confessions», Steak haché, n 41, 2001.

+ "La télévision à Timmins» et "Audrey Hepburn», Steak haché, n 42, 2001.

+ «Il y a un guépard», "Court métrage -1» et «Court métrage -2», Steak haché, n 43, 2001.

+ «Insérez titre ici», «Eric Clapton» et «Sous les plaines de la pleine Lune», Steak haché, n 44, 2001.

+ «Pourtant», José Acquelin, Sylvain Janneteau et Nuits urbaines, Brèches, Montréal, Groupe Plexus, 2001, p. 43-44.

+ «Mon chat» et «Ce courant de ton regard», Steak haché, n 45, 2002.

+ «L'acte de tristesse», «Rien d'trop beau » et «Une mouche meurt», Steak haché, n 46, 2002.

+ «Agnostic Angel», "Champlain Québec» et «Petit polar», Steak haché, n 47, 2002.

+ «Face-off» et «Une si jolie veuve», Steak haché, n 48, 2002.

+ «L'endroit», «Crépuscule» et «Tahini», Steak haché, n 49, 2002.

+ «Les petits ganglions», «Maquille la montagne» et «M. O.», Steak haché, n 50, 2002.

+ «Elle hait les hommes», "Poisson messager» et «Le long cool dude», Steak haché, n 51, 2002.

+ «Jump-start», «Wawa», «Un autre poème», Steak haché, n 52, 2002.

+ «On arrivera jamais à l'amour», «Montréal-Moncton-Montréal» et «V'là l'bon vent», Steak haché, n 53, 2002.

+ "Muffin», "Quand nos lèvres comme des lièvres» et "Chaque masque qu'elle porte», Steak haché, no 54, 2002.

+ «Carte postale» et «Tomahawk», Steak haché, n 55, 2002.

+ «Pourquoi aurais-je besoin» et «En attente», Steak haché, n 56, 2002.

+ «Varloppe» et «Le désir», Steak haché, n 57, 2003.

+ «Mantra de mentries», Steak haché, n 58, 2003.

+ «Hurlesang» et «Mémo sur papier blanc», Steak haché, n 59, 2003.

+ «Un soir à l'académie comme tout le monde», Steak haché, nº 60, 2003.

+ «Dead Ducks», Estuaire, n 118, juillet 2004, p. 38-47.

+ "Je me souviens d'une station wagon...", accompagné de onze traductions anglaises, une traduction allemande et une traduction portugaise, Ellipse, n 71,2004 , p. 16-31. 
+ «Le mot composé... », «Poème pour les parents d'Aimée», «L'effet de la pluie poussée par le vent sur les bâtiments», «La misère sexuelle», «La débarbouillette jaune» et «La pauvreté», Anne Peyrouse (dir.), Humour et poésie. 30 poètes québécois, Trois-Rivières, Écrits des Forges, 2004, p. 36-42.

+ «Jack Where's Jack», Estuaire, nº 122, mai 2005, p. 74-85.

+ «Inédit aux fins d'autoportrait», Lettres québécoises, n 119, automne 2005, p. 5-6.

+ "L'effet de la pluie poussée par le vent sur les bâtiments», Le Recherché, Journal de l'Association des étudiants des cycles supérieurs de Polytechnique, vol. XIX, n 3, 2005.

+ «La Belle Province», Liberté, vol. XLVIII, n² 2, mai 2006, p. 14-15.

+ «Teueikan», poème en français et en anglais en hommage à son ami, le poète Robert Dickson, Liaison, n 136, été 2007, p. 32.

+ «La chérie canadienne» et "Canadian Sweetheart», Marc Charron, Seymour Mayne et Christiane Melançon (dir.), Pluriel: une anthologie, des voix/An Anthology of Diverse Voices, Ottawa, Presses de l'Université d'Ottawa, 2008, p. 38-39.

+ «Jaco Pastorius», François Hébert (dir.), J'partirai. 100 poèmes québécois sur la mort, Outremont, Éditions du Passage, coll. «Les derniers mots», 2009, p. 98-99.

+ «Pour de vrai», Estuaire, n 145 , juin 2011, p. 34.

+ «Cinq inédits» [«la belle aux bois endormants...», «je me coupe la peau...», «une note...», «en l'année quelconque quèque....» et «je me sens un peu comme...»], Liberté, nº 299, printemps 2013, p. 63.

+ «Mises à jour», Les écrits, no 150, été 2014, p. 93-96.

+ «insérez titre ici», Estuaire, n 161, juin 2015, p. 43-50.

+ «Elle est sans terre», «Ô que», «Un ciel couleur de cayenne» et «Encore elle encore», Contrejour. Cahiers littéraires, $n^{\circ}$ 40, automne 2016.

+ «Mises à jour», L'Inconvénient, nº 69, été 2017, p. 56.

+ «Avant la science», Mathieu Arsenault, 10 ans, 11 poètes, 12 affiches, Montréal, Possibles éditions, 2017. (Recueil de douze poèmes-affiches publié par la librairie Le port de tête pour célébrer son dizième anniversaire.)

\section{Critiques culturelles et textes d'opinion}

+ «Québec vacation» et «Charlebois», The Eye Opener, vol. III, n 13, 1969, p. 5.

+ «Appendum», The Eye Opener, vol. III, no 14, 1969, p. 15.

+ «Beefheart», The Eye Opener, vol. III, n 32, 1970, p. 7.

+ «Aznavour. Not At Hist Best», The Eye Opener, vol. III, n³3, 1970, p. 10.

+ «Morrison Hotel. Hard Rock Cafe», The Eye Opener, vol. III, n 34, 1970, p. 9.

+ «The Longest Week», The Eye Opener, vol. IV, n 4, 1970, p. 10-11.

+ «Mother and I» (dessin) et «Highway 20 Visited», The Eye Opener, vol. IV, nº 6, 1970, p. 4 et 13 .

+ «Safe Talk» («micro trottoir»), The Eye Opener, vol. V, n 1, 1971, p. 11.

+ «Le cocon et le couteau», L'Express de Toronto, vol. III, n 35, 1978.

+ «La reine... symbole impotent», en réponse à l'éditorial «Keep the Queen» du Toronto Sun du $1^{\text {er }}$ septembre 1978, L'Express de Toronto, vol. III, nº 36, 1978.

+ «Notes d'un téléspectateur laïc (pour Gilles)», L'Express de Toronto, vol. III, n 37, 1978.

+ «Un journal hebdomadaire c'est quotidien», L'Express de Toronto, vol. III, n 38, 1978.

+ «Au festival des fumistes», L’Express de Toronto, vol. III, n 39, 1978. 
+ «Nous sommes là... malgré Davis... malgré Crombie... malgré tout», L'Express de Toronto, vol. III, $n^{\circ}$ 40, 1978.

+ «Une explication sans issue», L'Express de Toronto, vol. III, n 41, 1978.

+ «Video cab: un effort intelligent et passionné», L’Express de Toronto, vol. III, n ${ }^{\circ}$ 43, 1978.

+ «Pour combien de temps?» et «Georgie McDonald», L'Express de Toronto, vol. III, n 45, 1978.

+ «Franco-Ontariens, unissez-vous!», L’Express de Toronto, vol. III, n 51, 1978.

+ «L'acrobate administratif», «La rigueur de Hearst», «Le cinéma à Toronto » et «1939-1945», Réaction, vol. VIII, n³, 1980.

+ [Sans titre] (éditorial, critique de l'album triple Sandanista du groupe The Clash et Doc at the Radar Station de Captain Beefhearth and the Magic Band), Réaction, vol. VIII, n 6, 1981.

+ «Hélène», «Si j'étais un avion de guerre» et «Comme un refrain» (poèmes accompagnés d'un éditorial sur les difficultés de faire paraître un journal), Réaction, vol. VIII, nº 6, 1981.

+ [Sans titre] (notes sur l'avenir du Québec), L'élan d'Amérique, Éditions Mythologie urbaine, vol. XI, n³ 3, 1996.

\section{RÉCEPTION CRITIQUE ET PRINCIPALES ÉTUDES ${ }^{3}$}

A. Chapitres de livres

+ BÉLANGER, Louis, «Patrice Desbiens: au cœur des fictions sociales», Hédi Bouraoui (dir.), La littérature franco-ontarienne: état des lieux, Sudbury, Université Laurentienne, 2000, p. 197226. Article repris dans Ali Reguigui et Hédi Bouraoui (dir.), Perspectives sur la littérature franco-ontarienne, édition revue et augmentée, Sudbury, Prise de parole, coll. «Agora», 2007.

+ CHABOT, Marcel, «L'espace ontarien dans Sudbury de Patrice Desbiens», Roger Chemain et Arlette Chemain Degrange (dir.), Imaginaires francophones, Nice, Université Nice-SophiaAntipolis, 1996, p. 255-261.

+ DICKSON, Robert, «"Les cris et les crisse!” Relecture d'une certaine poésie identitaire francoontarienne», Lucie Hotte et Johanne Melançon (dir.), Thèmes et variations: regards sur la littérature franco-ontarienne, Sudbury, Prise de parole, coll. «Agora», 2005, p. 183-202.

+ HOTTE, Lucie, «Entre l'être et le paraître: l'altérité et l'identité dans deux textes francoontariens», Yvan G. Lepage et Robert Major (dir.), Croire à l'écriture. Études de littérature québécoise en hommage à Jean-Louis Major, Orléans, Éditions David, 2000, p. 163-178.

+ HOTTE, Lucie, «L'inscription de l'espace dans la poésie franco-ontarienne», Robert Yergeau (dir.), Itinéraires de la poésie. Enjeux actuels en Acadie, en Ontario et dans l'Ouest canadien, actes du colloque tenu à l'Université d'Ottawa les 14 et 15 mars 2003, Hearst, Le Nordir, coll. «RogerBernard», 2004, p. 99-111.

+ HOTTE, Lucie, «Identité collective et identité individuelle: le sujet poétique en poésie francoontarienne», Michael Brophy et Mary Gallagher (dir.), Sens et présence du sujet poétique. La poésie de la France et du monde francophone depuis 1980, New York, Rodopi, 2006, p. 169-179.

+ HOTTE, Lucie, «L'espace textuel de la fragmentation chez Patrice Desbiens», Carlo Lavoie (dir.), Lire du fragment: analyses et procédés littéraires, recueil de textes en partie issus du

3 Cette section de la bibliographie recense uniquement les textes qui traitent substantiellement de Patrice Desbiens et de son œuvre. Pour l'ensemble des publications concernant l'auteur, incluant celles qui se contentent de le mentionner, consulter la base de données BIBLIFO du Centre de recherche en civilisation canadienne-française, conçue par la Chaire de recherche sur les cultures et les littératures francophones du Canada de l'Université d'Ottawa. 
$15^{\text {e }}$ Colloque annuel de l'Association des professeurs des littératures québécoise et acadienne de l'Atlantique tenu à l'Université de l'Île-du-Prince-Édouard en octobre 2005, Québec, Nota bene, coll. «Terre américaine», 2008, p. 317-334.

+ HOTTE, Lucie, "Exil, migrance et nostalgie dans l'œuvre de Patrice Desbiens», Michael Brophy et Mary Gallagher (dir.), La migrance à l'œuvre: repérages esthétiques, éthiques et politiques, Berne, Peter Lang, coll. «Littérature de langue française», 2011, p. 37-52.

+ KARCH, Pierre-Paul, «Une prise de parole, oui; mais pour dire quoi? Analyse transactionnelle des principaux textes de quelques poètes franco-ontariens», Jules Tessier et Pierre-Louis Vaillancourt (dir.), Les autres littératures d'expression française en Amérique du Nord, Ottawa, Presses de l'Université d'Ottawa, coll. «Cahiers du Centre de recherche en civilisation canadienne-française», 1987, p. 47-62.

+ LAGACÉ, Francis, "Apprivoiser sa langue comme une belle étrangère. La minorité dans la minorité: le cas du poète franco-ontarien Patrice Desbiens», Christiane Albert (dir.), Francophonie et identités culturelles, Paris, Karthala, coll. «Lettres du Sud», 1999, p. 85-106.

+ LAMOUREUX, Myriam, "Le "récit-poème" chez Patrice Desbiens : transformations du genre lyrique», René Audet (dir.), Enjeux du contemporain. Études sur la littérature actuelle, Québec, Nota bene, coll. "Contemporanéités», 2009, p. 143-159.

+ LASSERRE, Elizabeth, "Identité et minorité dans l'écriture de Patrice Desbiens», Lucie Hotte (dir.), La problématique de l'identité dans la littérature francophone du Canada et d'ailleurs, actes du colloque organisé par les étudiants du Département des lettres françaises de l'Université d'Ottawa les 12 et 13 mai 1994, Ottawa, Le Nordir, 1994, p. 73-80.

+ LASSERRE, Elizabeth, «Contre-magie: l'effet de réel comme effet poétique dans l'œuvre de Patrice Desbiens», Ginette Adamson et Jean-Marc Gouanvic, Francophonie plurielle, actes du congrès mondial du Conseil international d'études francophones tenu à Casablanca (Maroc) du 10 au 17 juillet 1993, Casablanca/LaSalle, Eddif/Hurtubise HMH, 1995, p. 277-285.

+ LASSERRE, Elizabeth, «Un poète au seuil de l'écriture: l'exiguïté selon Patrice Desbiens», Lucie Hotte et François Ouellet (dir.), La littérature franco-ontarienne: enjeux esthétiques, actes du colloque tenu à l'Université McGill le 17 mai 1996, Ottawa, Le Nordir, 1996, p. 27-42.

+ LASSERRE, Elizabeth, «La littérature franco-ontarienne: ruptures et continuité», Hédi Bouraoui (dir.), La littérature franco-ontarienne: état des lieux, Sudbury, Université Laurentienne, 2000, p. 29-48.

+ LECLERC, Catherine, «Le colinguisme de l'exiguïté: L'homme invisible/The Invisible Man et Bloupe", Des langues en partage? Cohabitation du français et de l'anglais en littérature contemporaine, Montréal, XYZ éditeur, coll. «Théorie et littérature», 2010, p. 285-377.

+ LECLERC, Catherine et Nicole NOLETTE, «Pour ou contre la traduction: L'homme invisible/The Invisible Man de Patrice Desbiens», Arnaud Bernadet et Philippe Payen De La Galanderie (dir.), Traduire-écrire. Cultures, poétiques, anthropologie, Paris, ENS Éditions, coll. «Signes», 2014, p. 257-275.

+ MELANÇON, Johanne, «Une ville régionale, ouvrière et mythique: représentations de Sudbury dans la poésie franco-ontarienne», Anne-Yvonne Julien (dir.), Littératures québécoise et acadienne contemporaines: au prisme de la ville, avec la collaboration d'André Magord, Rennes, Presses universitaires de Rennes, coll. «Plurial», 2014, p. 151-164.

+ NEPVEU, Pierre, «Le complexe de Kalamazoo», Intérieurs du Nouveau Monde. Essais sur les littératures du Québec et des Amériques, Montréal, Boréal, coll. "Papiers collés», 1998, p. 266-294. 
+ NOLETTE, Nicole, «Le jeu de clignotements de l'homme invisible», Jouer la traduction. Théâtre et hétérolinguisme au Canada francophone, Ottawa, Presses de l'Université d'Ottawa, coll. «Regards sur la traduction», 2015, p. 151-169.

+ OUELLET, François, «La poésie ou la faillite de la posture paternelle: l'œuvre de Patrice Desbiens», Lucie Hotte et Johanne Melançon (dir.), Thèmes et variations : regards sur la littérature franco-ontarienne, Sudbury, Prise de parole, coll. «Agora», 2005, p. 203-225.

+ OUELLET, François, «Patrice Desbiens par lui-même: 1974-1995», Jacques Paquin (dir.), Nouveaux territoires de la poésie francophone au Canada, 1970-2000, Ottawa, Presses de l'Université d'Ottawa/CRCCF, coll. «Archives des lettres canadiennes», 2012, p. 235-265.

+ PARÉ, François, «L'indignation», «Le spectacle ritualisé de la disqualification», «Patrice Desbiens récité par Jacques Boulanger aux Beaux Dimanches», "Conscience et oubli: les deux misères de la parole franco-ontarienne» et «Le songe de Patrice Desbiens», Les littératures de l'exiguïté, Hearst, Le Nordir, coll. «Essai», 1994 [1992], p. 55-56, p. 63-64, p. 96-98, p. 123-135, p. 136-137.

+ PARÉ, François, «Patrice Desbiens dans les bras de sa mère», Le fantasme d'Escanaba, Québec, Nota bene/CEFAN, 2007, p. 87-105.

+ PUCCINI, Paola, «L'autotraduction responsable: Patrice Desbiens», Autotraduction et reconfiguration identitaire. Marco Micone, Madeleine Blais-Dahlem, Patrice Desbiens, Bologne, I Libri Di Emil, 2017, p. 111-129.

+ SIMARD, Mathieu, «Le conflit des genres. Classes sociales et classes de textes chez Patrice Desbiens et Jean Chicoine», Louis Bélanger (dir.), Les vingt-cinq ans de l'APLAQA. État des lieux et perspectives d'avenir, Moncton, Éditions Perce-Neige, coll. «Archipel/APLAQA», p. 105-116.

+ TESSIER, Jules, «De l'anglais comme élément esthétique à part entière chez trois poètes du Canada français: Charles Leblanc, Patrice Desbiens et Guy Arsenault», André Fauchon (dir.), La production culturelle en milieu minoritaire, Saint-Boniface, Centre d'études francocanadiennes de l'Ouest, 1994, p. 255-273.

+ WATTEYNE, Nathalie, "“Riveraine" de Jacques Brault et Un pépin de pomme sur un poêle à bois de Patrice Desbiens: présence de l'absente», Denise Brassard et Évelyne Gagnon (dir.), États de la présence. Les lieux d'inscription de la subjectivité dans la poésie québécoise actuelle, Montréal, XYZ éditeur, coll. «Théorie et littérature», 2010, p. 281-292.

+ WATTEYNE, Nathalie, "Les déclassés de Sudbury», Yvon Rivard et Sarah Rocheville (dir.), Figures de compassion, Montréal, Leméac, 2014, p. 115-130.

\section{B. Articles parus dans des revues scientifiques}

+ BOIVIN, Geneviève, «L'intime et malaisée poésie», Revue Chameaux, n 7, 2014, en ligne: https://revuechameaux.org/index.php/numeros/ecritures-de-lintime-au-masculin/lintime-etmalaisee-poesie/ (page consultée le 25 février 2019).

+ DICKSON, Robert, «L'espace à créer et l'espace qui reste», Revue du Nouvel-Ontario, nº 4, 1982, p. $45-80$.

+ DICKSON, Robert, «Autre, ailleurs et dépossédé. L'œuvre poétique de Patrice Desbiens», Revue de l'Université d'Ottawa, vol. LVI, nº 3, juillet-septembre 1986, p. 19-34.

+ DICKSON, Robert, "Moi e(s)t l'autre: quelques représentations de mutation identitaire en littérature franco-ontarienne», Francophonies d'Amérique, n 11, 2001, p. 77-89.

+ DOIRE, Nicolas, «Entre Sudbury et l'amour: l'évolution du mythe amoureux chez Patrice Desbiens", Lucie Hotte et Johanne Melançon (dir.), Thèmes et variations: regards sur la littérature franco-ontarienne, Sudbury, Prise de parole, coll. «Agora», 2005, p. 227-241. 
+ HÉBERT, François, «Dans la lune de Patrice Desbiens: de la tumeur à l'oreiller», Études canadiennes/Canadian Studies, n 64 , 2008, p. 195-204.

+ HOTTE, Lucie, «Artiste, animateur culturel ou médiateur culturel? Le rôle des artistes dans les communautés francophones du Canada», Minorités linguistiques et société, n 3, 2013, p. 7-18.

+ KILLEEN, Marie-Chantal, «La problématique du bilinguisme, Franco-Ontarian Style: L’homme invisible/The Invisible Man de Patrice Desbiens", Tangence, n 56, décembre 1997, p. 80-90.

+ LAGRANDEUR, Kathryn, "Dé-placements desbiensiens: langue(s), culture(s), et texte(s)», Revue Frontenac, nº 13, 1996, p. 51-57.

+ LASSERRE, Elizabeth, «Écriture mineure et expérience minoritaire: la rhétorique du quotidien chez Patrice Desbiens», Études françaises, vol. XXXIII, n 2, automne 1997, p. 63-76.

+ LECLERC, Catherine, «Bilinguisme officiel et traduction au Canada: les interprétations littéraires de Patrice Desbiens et de Jacques Brault/E. D. Blodgett», Meta. Journal des traducteurs/ Translators' Journal, vol. LIX, nº 3, décembre 2014, p. 494-516.

+ LÉGER, Richard, «L'espace comme lieu d'aliénation par le quotidien dans Sudbury (poèmes 1979-1985)", Revue du Nouvel-Ontario, n³1, 2006, p. 13-31.

+ MACDONELL, Alan, «Colonisation et poétique: Patrice Desbiens, poète franco-ontarien», Travaux de littérature, $\mathrm{n}^{\circ}$ 7, 1994, p. 367-378.

+ MAILHOT, Valérie, "Exiguïté et nord-américanité: la parole piégée dans Sudbury de Patrice Desbiens», DIRE (DIversités REcherches et Terrains), n 5, 2014, en ligne: http://epublications. unilim.fr/revues/dire/457 (page consultée le 25 février 2019).

+ MANGADA, Beatriz, "Tradiciones literarias franco-canadienses: el caso de Ontario», Anales de Filología Francesa, n 20, 2012, p. 155-172.

+ PARÉ, François, "Conscience et oubli: les deux misères de la parole franco-ontarienne», Revue du Nouvel-Ontario, nº 4, 1982, p. 89-102.

+ SIMARD, Mathieu, «Norme unilingue/Norme multilingue: revisiter le bilinguisme littéraire de l'écrivain franco-ontarien Patrice Desbiens», Continents manuscrits. Génétique des textes littéraires - Afrique, Caraibe, diaspora, n² 2, 2014, en ligne: http://journals.openedition.org/ coma/313 (page consultée le 25 février 2019).

+ SIMARD, Mathieu, «Pour une poétique de la non-coïncidence. Différence des langues, des voix et des points de vue dans l'œuvre de Patrice Desbiens», Fabula-LhT. Littérature, histoire, théorie, no 12 , mai 2014, en ligne: http://www.fabula.org/lht/12/simard.html (page consultée le 25 février 2019).

+ SIMARD, Mathieu, "Le "déplacement" dans Les cascadeurs de l'amour de Patrice Desbiens», Revue Frontenac, $\mathrm{n}^{\circ} 23,2015$, p. 40-50.

+ SIMARD, Mathieu, «Les porteuses de langues: perte et acquisition des connaissances linguistiques dans L'homme invisible/The Invisible Man», @nalyses. Revue de critique et de théorie littéraire, vol. X, n 1, hiver 2015, en ligne: https://uottawa.scholarsportal.info/ojs/ index.php/revue-analyses/article/view/1241 (page consultée le 25 février 2019).

+ SIMARD, Mathieu, «Roméo et/and Juliet. Les paysages amoureux de Patrice Desbiens», Temps zéro, no 10, 2015, en ligne: http://tempszero.contemporain.info/document1340 (page consultée le 25 février 2019).

+ TENNIER, Julie, «Ressemblance et étrangeté: l'altérité dans quelques œuvres francoontariennes», LittéRéalité, vol. XX, nº 1, printemps-été 2008, p. 59-73.

+ TESSIER, Jules, «Quand la déterritorialisation déschizophrénise ou De l'inclusion de l'anglais dans la littérature d'expression française hors Québec», Traduction, terminologie, rédaction. Études sur le texte et ses transformations, vol. IX, nº 1, 1996, p. 177-209. 
+ WATTEYNE, Nathalie, «Desbiens, le regard du poète tourné vers le visage quelconque», Études québécoises. Revue internationale de l'Association coréenne d'études québécoises [Séoul], nº 5 , 2011, p. 91-110.

+ YERGEAU, Robert, «La poésie franco-ontarienne: les lieux de la dépossession», Francophonies d'Amérique, no 1, 1991, p. 7-13.

\section{Articles parus dans des journaux et des magazines culturels}

+ [S. n.], «Patrice Desbiens», Cahier supplémentaire du Voyageur, 9 octobre 1985, p. 9 b.

+ ARSENAULT, Mathieu, «Ruralité trash», Liberté, vol. LIII, n 3, avril 2012, p. 38-47.

+ ASSELIN, Olivier, «La Souche. Je suis poète», Liaison, n 16, juin 1981, p. 9. [Compte rendu des poèmes parus dans La Souche en 1981. Voir la section I-C de la présente bibliographie.]

+ BAILLARGEON, Normand, «Patrice Desbiens. La tendresse comme seule adresse. Le poète franco-ontarien a surmonté la misère de l'instabilité identitaire», Le Devoir, 11 mai 1998, p. B1.

+ BOISVERT, Yves, «Patrice le minimal», Lettres québécoises, n 119, automne 2005, p. 11-12.

+ CARMICHAEL, Harold, «More than a Business», Sudbury Star, 13 novembre 2004, p. B1.

+ CHAREST, Rémy, "Patrice Desbiens et le chemin de la route», Voir, 9 au 15 avril 1992, [s. p.].

+ COOK, Margaret Michèle, «Les périples de Patrice Desbiens», Liaison, nº 150, hiver 2010-2011, p. 58-59.

+ DEMERS, Véronique, «La poésie jazzée de Patrice Desbiens», Québec Hebdo, 19 mai 2010.

+ DUMAS, Ėve, «Patrice Desbiens, poète jour et nuit», La Presse, 16 août 2003, p. D6.

+ FORTIN, André, "Vision de Jack», Nuit blanche, n 30, décembre 1987-janvier 1988, p. 32-36.

+ GAGNON, Paulette, «Patrice Desbiens: tout a commencé ici», Liaison, n 84, novembre 1995, p. 14-16.

+ GAUTHIER, Stéphane, «Écrire avec des allumettes sur du papier sablé», Le Voyageur, 21 novembre 2007, p. 21.

+ HAENTJENS, Brigitte, «Patrice Desbiens, un grand écrivain. Son ironie protège du désespoir», Liaison, $n^{\circ}$ 40, automne 1986, p. 5.

+ HAMELIN, Louis, «Un super-héros dépareillé: Pat-Man», Liaison, no 95, janvier 1998, p. 19-21.

+ HÉBERT, Catherine, "Fiction pulpeuse», Voir, vol. XVII, n 33, 21 août 2003, p. 26.

+ LAMOTHE, Maurice, «La chanson francophone contemporaine des Amériques: quelques cas types hors Québec, de Calixte Duguay à Isabelle Longnus », Québec français, nº 154, été 2009, p. $42-53$.

+ LAROSE, Karim et Jean-Philippe WARREN, «Terra incognita», Liberté, n 299, printemps 2013, p. 14-18.

+ LASSERRE, Elizabeth, "Patrice Desbiens: "Je suis le franco-ontarien"», Nuit blanche, $\mathrm{n}^{\circ}$ 62, hiver 1995-1996, p. 64-68.

+ MACINA, Michel, «Patrice Desbiens. Un cri bloqué dans la gorge», Liaison, n 29, hiver 19831984, p. 25-27.

+ MONTPETIT, Caroline, "Écrivains francophones hors Québec. Seuls et pourtant si près», Le Devoir, 13 novembre 1999, p. D8.

+ OUELLET, François, «Écrivains franco-ontariens», Québec français, no 174, 2015, p. 59-60.

+ POIRIER, Alain, «À la recherche de l'homme invisible. Quelques détours pour voir du vrai monde», Liaison, n 66, mars 1992, p. 38-39. 
+ STEIN, Janice, «Gut Feelings Jotted down Day after Drunken Day», Northern Life, 7 novembre 1979, p. 2.

+ SYLVESTRE, Paul-François, «Il y a 55 ans: naissance du poète Patrice Desbiens», L'Express, 11 au 17 mars 2003, p. 3.

\section{Mémoires et thèses}

+ BAZINET, Valérie, Figures d'altérité transformatrice: l'Autre chez Victor-Lévy Beaulieu, Larry Tremblay, Dany Laferrière et Patrice Desbiens, mémoire de maîtrise, Montréal, Université Concordia, 2010, $111 \mathrm{f}$.

+ BLAIS, Mathieu, La conquête du quotidien dans Sudbury de Patrice Desbiens, suivie du recueil de poèmes Voracités, mémoire de maîtrise, Sherbrooke, Université de Sherbrooke, 2007, 144 f.

+ BOISVERT, Josée, L'anglais comme élément esthétique dans l'œuvre de Patrice Desbiens, mémoire de maîtrise, Ottawa, Université d'Ottawa, 1998, 145 f.

+ DAIGLE-ROY, Alice, Expérience précaire du monde et représentation de la poésie dans La fissure de la fiction et Désâmé de Patrice Desbiens, mémoire de maîtrise, Montréal, Université de Montréal, 2015, $127 \mathrm{f}$.

+ FILTEAU, Vincent, Le cauchemar canadien: survivance et témoignage dans l'Amérique postindustrielle chez Patrice Desbiens et Benoît Jutras, mémoire de maîtrise, Université du Québec à Montréal, 2017, 133 f.

+ FRÉCHETTE, Andrée-Anne, Réflexions sur le repli identitaire et les stratégies d'ouverture dans les littératures francophones du Nord, suivi de Grand langue, mémoire de maîtrise, Trois-Rivières, Université du Québec à Trois-Rivières, 2013, 154 f.

+ GAUTHIER, Stéphane, Lectures de soi : la construction et la représentation de l'identité dans quatre récits franco-ontariens contemporains (1981-1991), mémoire de maîtrise, Sherbrooke, Université de Sherbrooke, 1998, $280 \mathrm{f}$.

+ JOANNETTE, Karen, La métaphore et la comparaison ironiques dans quatre recueils de Patrice Desbiens, mémoire de maîtrise, Montréal, Université McGill, 2015, 112 f.

+ KIROUAC MASSICOTTE, Isabelle, Des mines littéraires : étude chronotopique de l'imaginaire minier dans les littératures abitibienne et franco-ontarienne, thèse de doctorat, Université d'Ottawa, 2016, $298 \mathrm{f}$.

+ LAMOUREUX, Myriam, Une prise de parole sur la langue. L'ambivalence générique dans l'écriture poétique de Gaston Miron et de Patrice Desbiens, mémoire de maîtrise, Québec, Université Laval, 2008, $124 \mathrm{f}$.

+ LAPALME, Julie, L'individu hypermoderne dans trois œuvres franco-ontariennes: une lecture d'André Paiement, de Patrice Desbiens et de Michel Ouellette, mémoire de maîtrise, Montréal, Université Concordia, 2008, 227 f.

+ LASSERRE, Elizabeth, Aspects de la néo-stylistique: étude des poèmes de Patrice Desbiens, thèse de doctorat, Toronto, University of Toronto, 1996, $353 \mathrm{f}$.

+ MARTINEAU, Julien, C'est ici que le verbe habiter s'est déchiré; suivi de Sudbury: l'habitabilité de la poésie chez Patrice Desbiens, mémoire de maîtrise, Montréal, Université de Montréal, 2011, 200 f.

+ MOULTON, Lori-Ann, Écritures minoritaires de l'exil: la nouvelle génération franco-ontarienne, mémoire de maîtrise, Hamilton, Université McMaster, 2004, 95 f.

+ PROULX, Marie-Pierre, La poésie de Patrice Desbiens à l'épreuve de la scène: adaptation textuelle et scénique de L'homme invisible/The Invisible Man, mémoire de maîtrise, Ottawa, Université d'Ottawa, 2012, $158 \mathrm{f}$. 
+ SIMARD, Mathieu, La poétique bilingue de Patrice Desbiens, mémoire de maîtrise, Montréal, Université McGill, 2013, 106 f.

+ SYLVAIN, Véronique, Au nord du Nord, au nord de soi, au nord de l'Autre... Une analyse du thème du Nord dans Décalage de Patrice Desbiens et dans L'espace éclaté de Pierre Albert, Ottawa, Université d'Ottawa, 2012, 154 f.

+ VAILLANCOURT, Danica, Problème d'expression: l'alternance codique et ses retombées sur l'identité individuelle et collective. Étude d'un corpus littéraire franco-ontarien et acadien, mémoire de maîtrise, Waterloo, Université de Waterloo, 2007, 102 f.

\section{E. Préfaces, entrées de dictionnaire et notices e n c y c l opédiques}

+ DICKSON, Robert, «En guise d'introduction/A Word of Introduction», Patrice Desbiens, L'homme invisible/The Invisible Man, Sudbury/Moonbeam, Prise de parole/Penumbra Press, 1981. Repris dans L'homme invisible/The Invisible Man, suivi de Les cascadeurs de l'amour, Sudbury, Prise de parole, 1997, p. 6-9.

+ HOTTE, Lucie, «DESBIENS, Patrice», Corinne Blanchaud (dir.), Dictionnaire des écrivains francophones classiques. Belgique, Canada, Québec, Luxembourg, Suisse romande, Paris, Honoré Champion, coll. «Champion les dictionnaires», 2013, p. 159-161.

+ LARIVIÈRE, Jean Marc, «Scénario pour une préface fissurée», Patrice Desbiens, Poèmes anglais, suivi de Le pays de personne, suivi de La fissure de la fiction, Sudbury, Prise de parole, coll. «BCF. Bibliothèque canadienne-française», 2010, p. 5-23.

+ MELANÇON, Johanne, «Relire L'homme invisible/The Invisible Man et Les cascadeurs de l'amour", Patrice Desbiens, L'homme invisible/The Invisible Man, Sudbury, Prise de parole, coll. «BCF. Bibliothèque canadienne-française», 2008, p. 5-15.

+ PARÉ, François, «Amour ambulance», «Les cascadeurs de l'amour», «Les conséquences de la vie», «Dans l'après-midi cardiaque», «L'espace qui reste», «L'homme invisible/The Invisible Man», «Ici», «Poèmes anglais» et «Sudbury», Gaétan Gervais et Jean-Pierre Pichette (dir.), Dictionnaire des écrits de l'Ontario français: 1613-1993, Ottawa, Presses de l'Université d'Ottawa, 2010, p. 35, 131, 190, 222, 310, 415, 423, 673 et 823-824.

+ SIMARD, Mathieu, «Patrice Desbiens: L'homme invisible/The Invisible Man», The Literary Encyclopedia, vol. 3.1.4, 2015, en ligne: https://www.litencyc.com/php/sworks. php?rec=true\&UID=35662 (page consultée le 25 février 2019).

\section{F. Comptes rendus ${ }^{4}$}

\section{Ici ( 1974$)$}

+ HAECK, Philippe, «La critique sociale de Desbiens et Toupin», Le Devoir, 7 juin 1975, p. 17.

+ ROYER, Jean, «Poèmes du contre-quotidien», Le Soleil, 26 juillet 1975, p. C6.

4 Lorsqu'un compte rendu porte sur plus d'un texte, il est placé dans la section consacrée à l'ouvrage paru le premier, et suivi d'une indication spéciale entre crochets. Noter que la présente liste n'inclut pas les comptes rendus des adaptations théâtrales de textes de Patrice Desbiens. 


\section{Les conséquences de la vie (1977)}

+ BEAULIEU, Michel, «Quelques poètes d'outre-frontière», Le livre d'ici, vol. IV, n 34, 30 mai 1979, p. 1. Repris dans L'Express de Toronto, 8 juin 1979, p. 12, et dans Le Nord de Hearst, 27 juin 1979, p. K11.

+ GAY, Paul, «Patrice Desbiens le surréaliste», Le Droit, 20 septembre 1980, p. 18. [Sur Les conséquences de la vie et L'espace qui reste.]

+ PRATTE, Jean-Paul, «Les conséquences de l'écriture», Le Voyageur, 8 juin 1977, supplément culturel, p. 42.

\section{L'espace qui reste (1979)}

+ ASSELIN, Olivier, «Le cancer poétique de Patrice Desbiens: L'espace qui reste», Liaison, vol. III, n 9, avril 1980, p. 32.

+ BEAUlIEU, Michel, «Un nouveau recueil de poèmes de Patrice Desbiens», Le Nord, 10 octobre 1979, p. H24. Repris dans Le Voyageur, 10 octobre 1979.

+ GIGUÈRE, Richard, «En d'autres lieux (de poésie)», Lettres québécoises, n 17, printemps 1980, p. 30-34.

+ MOISAN, Clément, «Alexandre Amprimoz, 10/11; Gaston Tremblay, Souvenances; Patrice Desbiens, L'espace qui reste, Prise de parole», Livres et auteurs québécois 1979, 1980, p. $92-94$.

\section{L'homme invisible/The Invisible Man et L'homme invisible/} The Invisible Man, suivi de Les cascadeurs de l'amour (1981, 1997,2001 et 2008 )

+ (1981) AUBERT, Rosemary, «New Stars in the Galaxy of Canadian Poetry», Quill \& Quire, vol. XLVIII, n 4, avril 1982, p. 30.

+ (1997) BARRAS, Nancy-Gaëlle, «L'homme invisible/The Invisible Man. Les cascadeurs de l'amour", L'orignal déchaîné, 3 décembre 1997, p. 6.

+ (1981) BEAULIEU, Michel, «Poète de Timmins: L'homme invisible de Patrice Desbiens», Livre d'ici, vol. VII, n 38, 23 juin 1982, p. 10.

+ (1981) BERGERON, François, «Les Franco-Ontariens invisibles? Dans son nouveau livre, Patrice Desbiens récupère le sens propre de cette image populaire», L'Express de Toronto, vol. VII, $\mathrm{n}^{\circ} 3$, du 26 janvier au $1^{\text {er }}$ février 1982, p. 1.

+ (1981) D’ALFONSO, Antonio, "Patrice Desbiens. L'homme invisible/The Invisible Man», Nos livres, vol. XIII, avril 1982, p. 155.

+ (1981) DALLAIRE, Michel, «Le trait d'union, une réalité en soi», Liaison, n 23, aoûtseptembre 1982, p. 43-44.

+ (1997) LASSERRE, Elizabeth, «Écrits franco-ontariens», Canadian Literature, nº 164, printemps 2000, p. 146-147. [Sur L'homme invisible/The Invisible Man, suivi de Les cascadeurs de l'amour et La fissure de la fiction.]

+ (1981) LIDDLE, Michel, «Le voyage dans la littérature du Nouvel-Ontario », Liaison, n 39, juin 1986, p. 22-24.

+ (1981) PUNCH, Katherine, «The Invisible Man by Patrice Desbiens», The Sault Star, $1^{\mathrm{er}}$ mai 1982, p. 14.

+ (1981) RENAUD, Normand, «Romans et nouvelles d'Acadie, d'Ontario et du Manitoba», Livres et auteurs québécois 1982, 1983, p. 23-26. 
+ (1981) RENAUD, Normand, «Patrice Desbiens. L'homme invisible/The Invisible Man», Livres et auteurs québécois 1982, 1983, p. 43-44.

+ (1981) ROYER, Jean, «La littérature franco-ontarienne. Prendre la parole pour ne pas disparaître», Le Devoir, 19 février 1983, p. 19 et 36.

+ (1981) SALESSE, Michèle, «L'homme invisible/The Invisible Man, récit/story de Patrice Desbiens», Lettres québécoises, n 26, été 1982, p. 79-80.

+ (1981) STUEWE, Paul, «Foreign Affairs: From National Turmoil in Brazil to Unreal World of Official Czech Literature», Books in Canada, août-septembre 1982, p. 34-35.

+ (1981) SYLVESTRE, Paul-François, "L'homme invisible/The Invisible Man», Le temps de lire, avril 1982.

\section{Sudbury: textes 1981-1983 (1983)}

+ DICKSON, Robert, «Sudbury», Revue du Nouvel-Ontario, n 5, 1983, p. 163-165.

+ KARCH, Pierre-Paul, «Sudbury. Réflexions utiles d'un drame personnel», L'Express, 9 au 15 août 1983.

+ LACOMBE, François, "Sudbury, “... où la communication est difficile" ", Liaison, n 28, septembre 1983, p. 63.

+ LÉPINE, Stéphane, «Desbiens (Patrice). Sudbury», Nos livres, vol. XIV, octobre 1983, p. 17-18.

+ YERGEAU, Robert, «La traversée du réel. Patrice Desbiens: le Bukowski nordique», Lettres québécoises, nº 36, hiver 1984-1985, p. 34-35.

\section{Dans l'après-midi cardiaque (1985)}

+ [S. n.], «Nouveauté à Prise de parole», Le Voyageur, 9 octobre 1985, p. 116.

+ BÉLANGER, Paul, «Dans l'après-midi cardiaque», Nuit blanche, nº 24, juillet-aoûtseptembre 1986, p. 5-6.

+ BENSON, Mark, «Maniaques dépressifs», Canadian Literature, nº 112, printemps 1987, p. 138-141.

+ D’ALFONSO, Antonio, «Desbiens (Patrice). Dans l'après-midi cardiaque», Nos livres, 1986, nº 6439.

+ DUMONT, Jean, «Desbiens, Patrice, Dans l'après-midi cardiaque, Sudbury, Prise de parole, 1985, 77 p. ", L'Apropos, vol. IV, nº 1, 1986, p. 120-121.

+ NEPVEU, Pierre, «Sudbury Blues. Dans l'après-midi cardiaque de Patrice Desbiens», Spirale, nº 62, été 1986, p. 22.

+ PARÉ, François, «Dans l'après-midi cardiaque de Patrice Desbiens. La voix de l'homme qui a peur», Le Droit, 12 juillet 1986, p. 36.

+ YERGEAU, Robert, «Dans l'après-midi cardiaque: Desbiens joue à être Desbiens», Liaison, n³8, printemps 1986, p. 56.

\section{Les cascadeurs de l'amour (1987)}

+ ALBERT, Pierre, "'Les cascadeurs de l'amour": sec, caustique mais percutant», Le Nord, 14 octobre 1987, p. 9.

+ COURCHESNE, Michel, "Patrice Desbiens. Les cascadeurs de l'amour», Revue du NouvelOntario, n 9 , 1987, p. 146-147.

+ DUMONT, Jean, «Quand Paillasse devient Cascadeur», Liaison, n 46, printemps 1988, p. 52.

+ LAURIN, Michel, «Prise de parole lance trois nouveaux titres», Le Nouvel Ontarien, 2 octobre 1987, p. 3. 
+ LEDUC, André, "Les cascadeurs de l'amour de Patrice Desbiens. Quand la conscience détachée observe la conscience engagée se débattre», Le Droit, samedi 10 octobre 1987, p. 46.

+ WILLIAMS, Deloris, «Il est écrit», Canadian Literature, nºs 122-123, automne-hiver 1989, p. 263-265.

\section{Poèmes anglais ( 1988 )}

+ [S. n.], "Prise de parole releases two books by French authors», The Sudbury Star, 19 octobre 1988, p. 24.

+ FELX, Jocelyne, «Les langues d'Ésope», Lettres québécoises, n 53, printemps 1989, p. 33-34.

+ GAGNON, Liette, «Poèmes anglais», Livres, vol. II, nº 8, 1988, p. 24.

+ JIMENEZ, Yolande, "Dualité incertaine d'une conscience», Liaison, n 51, mars-avril 1989, p. 21.

+ POIRIER, Jacques, "Desbiens éprouve des "difficultés temporaires"», Le Nord, 5 octobre 1988, p. HA6.

\section{Amour ambulance (1989)}

+ DICKSON, Robert, «L'éloignement pour survivre», Nuit blanche, n 62, hiver 1995-1996, p. 68.

+ LIDDLE, Michel, «Poétique/politique de la détresse», Liaison, n 57, mai 1990, p. 21.

\section{Un pépin de pomme sur un poêle à bois (1995)}

+ BEAUDOIN, Daniel-Louis, «Patrice Desbiens. Un pépin de pomme sur un poêle à bois», Mœbius, $\mathrm{n}^{\circ}$ 66, hiver 1996, p. 148-151.

+ CORRIVEAU, Hugues, «Et souigne la baquaise dans le coin de la boîte à bois!», Lettres québécoises, nº 80, hiver 1995, p. 37-38.

+ LASSERRE, Elizabeth, «Desbiens est de retour, Desbiens est en forme», Liaison, n 83 , septembre 1995, p. 41.

+ OUELLET, François, «Patrice Desbiens, Un pépin de pomme sur un poêle à bois», Nuit blanche, n 62, hiver 1995-1996, p. 66-67.

\section{La fissure de la fiction (1997)}

+ BARRAS, Nancy-Gaëlle, "La fissure de la fiction», L'orignal déchaîné, 3 décembre 1997, p. 6.

+ BARRIÈRE, Caroline, "Littérature franco-ontarienne: les auteurs d'ici sont prolifiques», Le Droit, 20 septembre 1997, p. A13.

+ BERTIN, Raymond, «Patrice Desbiens», Voir, vol. XII, n 51, 24 décembre 1998, p. 12.

+ CANTIN, David, «Désespoir ironique», Le Devoir, 17 janvier 1998, p. D3. [Sur La fissure de la fiction et L'effet de la pluie poussée par le vent sur les bâtiments (édition de 1997).]

+ CHAMBERLAND, Roger, «Poésie», University of Toronto Quarterly, vol. LXVIII, nº 1, hiver 1998-1999, p. 38-58.

+ CÔTÉ, Gilles, «Patrice Desbiens, La fissure de la fiction», Nuit blanche, n 70, printemps 1998, p. 11-12.

+ FELX, Jocelyne, «Villes subjectives. Entre l'intime et l'universel, l'éternité a aussi des racines», Lettres québécoises, n 90, été 1998, p. 33-34.

+ OUELLET, François, «La béance du quotidien», Liaison, n 94, novembre 1997, p. 27. 


\section{L'effet de la pluie poussée par le vent sur les bâtiments}

(1999)

+ BÉLANGER, Louis, «Patrice Desbiens: des mots, des choses», Spirale, n 174 , septembreoctobre 2000, p. 13. [Sur L'effet de la pluie poussée par le vent sur les bâtiments, Rouleaux de printemps et Patrice Desbiens et les Moyens du bord.]

+ BERTIN, Raymond, «Nicole Brossard/Patrice Desbiens», Voir, vol. XIII, n² 22, 3 juin 1999, p. 98.

+ CÔTÉ, Gilles, «Patrice Desbiens, L'effet de la pluie poussée par le vent sur les bâtiments», Nuit blanche, n 76 , automne 1999, p. 17.

\section{Rouleaux de printemps (1999)}

+ BARRIĖRE, Caroline, "Une bonne récolte. Livres d'ici», Le Droit, 4 septembre 1999, p. A15.

+ BERTIN, Raymond, «Rouleaux de printemps», Voir, 25 novembre 1999, [s. p.].

+ CHAMBERLAND, Roger, «Poésie», University of Toronto Quarterly, vol. LXX, n 1, hiver 20002001, p. 39-54.

+ CÔTÉ, Gilles, «Rouleaux de printemps», Nuit blanche, nº 78, printemps 2000, p. 18.

+ FELX, Jocelyne, «Trois visions du quotidien», Lettres québécoises, n 98, été 2000, p. 43-44.

+ OLSCAMP, Marcel, «Un poète traqué», Liaison, n 108, septembre 2000, p. 41.

+ ROCHAT, Denise, "Poète de l'entre-deux-mondes», Canadian Literature, n ${ }^{\circ} 176$, printemps 2003, p. 143.

\section{Patrice Desbiens et les Moyens du bord (1999)}

+ BRUNET, Alain, «La poésie et les moyens du bord», La Presse, 23 octobre 1999, p. D10.

+ CANTIN, David, «Le poète comme improvisateur», Le Devoir, 13 novembre 1999, p. D26.

+ DENIS, Dominique, «Une joyeuse beurrée de poésie», L'Express, 30 novembre au 6 décembre 1999, p. 12.

+ LEMYRE, Marc, «Patrice Desbiens et les Moyens du bord: solide échafaud», Liaison, n 105 , février 2000, p. 23.

\section{Bleu comme un feu ( 2001$)$}

+ BÉLANGER, Louis, «Poètes de l'incantation», Liaison, n 114, printemps 2002, p. 54-55.

+ CÔTÉ, Christian, «D'été et de poésie», Le Droit, 14 juillet 2001, p. A15.

+ DULAC, Suzette, «Bleu comme un feu de Patrice Desbiens», L'Express, 22 au 28 mai 2001, p. 10.

+ FELX, Jocelyne, "Plongeon dans l'amour», Lettres québécoises, n 104, hiver 2001, p. 43-44.

+ HOTTE, Lucie, «Bleu comme un feu de Patrice Desbiens», Nuit blanche, n 85, hiver 2001-2002, en ligne: http://www.nuitblanche.com/commentaire-lecture/bleu-comme-un-feu/ (page consultée le 25 février 2019).

\section{Hennissements ( 2002 )}

+ BÉLANGER, Louis, «Rien d'autre à faire», Liaison, n 118, printemps 2003, p. 70.

+ BISSONNETTE, Thierry, «Diagonales du fou. Une version remaniée et augmentée d'un des premiers recueils de Patrice Desbiens", Le Devoir, 22 mars 2003, p. F3.

+ CORMIER, Pénélope, "Vivre condamné», L'Acadie Nouvelle, 14 février 2003, p. ACCENT 2.

+ CÔTÉ, Gilles, «Hennissements», Nuit blanche, no 91, été 2003, en ligne: http://www. nuitblanche.com/commentaire-lecture/hennissements/ (page consultée le 25 février 2019).

+ LAPIERRE, Michel, «Le drapeau du ciel», Ici, 10 avril 2003, p. 32. 
+ OLSCAMP, Marcel, «Lettres canadiennes 2002. Poésie», University of Toronto Quarterly, nº 1 , hiver 2003-2004, p. 439-460.

+ PAQUIN, Jacques, "Chroniques de poètes», Lettres québécoises, n 111, automne 2003, p. 39-40.

+ PARÉ, François, «Scènes d'automne», Canadian Literature, n ${ }^{183}$, hiver 2004, p. 120-122.

\section{Grosse guitare rouge (2004)}

+ BEAUDET, Simon-Pierre, «Grosse guitare rouge», Le libraire, novembre 2004, p. 16.

+ CÔTÉ, Gilles, «Grosse guitare rouge», Nuit blanche, n 98, printemps 2005, p. 19.

+ DENIS, Dominique, «L'amour démaquillé», Liaison, no 125, hiver 2004-2005, p. 51.

+ PARÉ, François, «Du gramophone au livre hybride. La voix des poètes», Liaison, n 129, 2005, p. 64-67.

+ PARÉ, François, «Le mythe incomparable du pauvre», Canadian Literature, n 190, automne 2006, p. 155-156. [Sur Grosse guitare rouge et Désâmé.]

\section{Désâmé ( 2005$)$}

+ DOIRE, Nicolas, "Désâmé, de Patrice Desbiens: quand la mort se rapproche du quotidien", Liaison, n 128, automne 2005, p. 56.

+ LANDRY, Gabriel, «Less is more», Voix et Images, vol. XXX, n³ 3, printemps 2005, p. 170.

\section{En temps et lieux 1,2, 3 et En temps et lieux: les cahiers} complets (2007,2008,2009 et 2017)

+ [S. n.], «Une merveilleuse banalité», Nuit blanche, $\mathrm{n}^{\circ}$ 110, printemps 2008, p. 7.

+ [S. n.], «Poésie franco-ontarienne», Nuit blanche, nº 113, hiver 2009, p. 6.

+ CORRIVEAU, Hugues, «Présence de Patrice Desbiens», Le Devoir, 23 et 24 février 2008, p. F4.

+ CÔTÉ, Gilles, «Patrice Desbiens, En temps et lieux», Nuit blanche, nº 111, été 2008, p. 29.

+ GIRARD, Christian, «Le dernier cahier», Le libraire, décembre 2009-janvier 2010, p. 15.

+ MARCHAMPS, Guy et Clément MORIN, «En temps et lieux», Le libraire, n 45, février-mars 2008.

+ PAQUIN, Jacques, «Petites choses», Lettres québécoises, n 134, été 2009, p. 44.

+ RHÉAUME, Stéphanie, «Pleins feux sur la Nouvelle Scène. Le théâtre francophone propose trois pièces pour terminer l'année en beauté», L'Express, 23 octobre 2006.

+ SIMON, Philippe, «Les instants saisis de Patrice Desbiens», Ouest-France. Ancenis CulturesMagazine, 9 octobre 2017, p. Ancenis_22.

\section{Décalage ( 2008 )}

+ GAGNON, Daniel, «Poésie», University of Toronto Quarterly, vol. LXXIX, n 1, hiver 2010, p. 37-64.

+ LECLERC, Rachel, «Unité de lieu, unité de temps», Lettres québécoises, n 135, automne 2009, p. 39.

+ LEMELIN, Pierre, «La poésie imagée telle un scrapbook», Le Voyageur, 5 novembre 2008.

+ MACDONELL, Alan, «Décalage», Cahiers franco-canadiens de l'Ouest, vol. XX, nº 1-2, 2008, p. 187-190.

+ MALAVOY-RACINE, Tristan, "Rentrée culturelle», Voir, vol. XXII, n 35, 4 septembre 2008.

+ NOLETTE, Nicole, «Quelques décalages», Canadian Literature, nº 207, hiver 2010, p. 127-128. 
+ PARÉ, François, «Les traîneries de la mémoire», Liaison, n 143, printemps 2009, p. 62-63.

21. Poèmes anglais, suivi de Le pays de personne, suivi de La fissure de la fiction (2010)

+ COOK, Margaret Michèle, «Les périples de Patrice Desbiens», Liaison, n 150, hiver 2010-2011, p. 58-59.

+ CORRIVEAU, Hugues, «Poésie. Des poèmes en skidoo?», Le Devoir, 23 janvier 2010, p. F4.

+ CÔTÉ, Gilles, "Patrice Desbiens. Poèmes anglais, Le pays de personne, La fissure de la fiction", Nuit blanche, n 121, hiver 2011, p. 16-31.

+ GAGNON, Daniel, «Poésie 2010», University of Toronto Quartely, vol. LXXXI, n³ 3, été 2012, p. 376-403.

+ LEPAGE, Élise, «Permanence de la fissure», Canadian Literature, n 212, printemps 2012, p. 145.

+ PAQUIN, Jacques, «"Je suis un Canadien erreur”», Lettres québécoises, n 141, printemps 2011, p. $40-41$.

\section{Pour de vrai (2011)}

+ SIMARD, Mathieu, "La vérité, toute la vérité», Salon double. Observatoire de la littérature contemporaine, 2013, en ligne: http://salondouble.contemporain.info/la-verite-toute-la-verite (page consultée le 25 février 2019).

\section{Sudbury (poèmes 1979-1985) (2013)}

+ CORRIVEAU, Hugues, «Horizon dégagé», Le Devoir, 31 août et $1^{\text {er }}$ septembre 2013, p. F4.

+ FALQ, Armand, «Sudbury (poèmes 1979-1985)», Voix plurielles, vol. XI, n 1, 2014, p. 345-346.

+ LECLERC, Rachel, «Patrice Desbiens, "citoyen de cette folie"», Lettres québécoises, nº 155, automne 2014, p. 46-47.

+ LESSARD, Valérie, «Des tonnes de feuilles d'ici pour la rentrée», Le Droit, 7 septembre 2013, p. A14-A15.

+ PETERSON, Michel, «L'impensable, l'ineffable... », Liaison, n 114, printemps 2002, p. 20-24.

+ TARDIF, Dominic, «Le livre parfait pour brailler dans sa bière», La Nouvelle, 22 avril 2015, p. N11.

\section{Les abats du jour (2013)}

+ [S. n.], «Les mots de Desbiens», Nuit blanche, n 132, automne 2013, p. 4.

+ AUSSANT, Éric, «Les abats du jour», Journal Métro, 12 juin 2013.

+ CORRIVEAU, Hugues, «Le déroutant Patrice Desbiens», Le Devoir, 24 et 25 août 2015, p. F5.

\section{Vallée des cicatrices (2015)}

+ ELAWANI, Ralph, "Accoucher d'un cœur par césarienne», Liberté, n 313, automne 2016, p. 67.

+ OUELLET, François, "Vallée des cicatrices», Nuit blanche, n 142, hiver 2016, p. 24-32.

+ TARDIF, Dominic, «Patrice Desbiens: partout et nulle part», Le Devoir, samedi 17 et dimanche 18 octobre 2015, p. F1, en ligne: http://www.ledevoir.com/culture/livres/452696/poesiepatrice-desbiens-partout-et-nulle-part (page consultée le 25 février 2019).

\section{Le quotidien du poète (2016)}

+ [S. n.], «L'ordinaire extraordinaire», Nuit blanche, no 142, hiver 2016, p. 12. 
+ CHARPENTIER, Marie, «Desbiens, Patrice. Le quotidien du poète», Voix plurielles, vol. XII, nº 2, 2016, p. 200-201.

+ DULUDE, Sébastien, «Le plus important», Lettres québécoises, n 163, automne 2016, p. 46.

+ LESSARD, Valérie, "Le quotidien du poète», Le Droit, 2 avril 2016, p. A15.

+ SIMARD, Mathieu, «Leçons de poésie, leçons de la poésie», Liaison, nº 172, été 2016, p. 54.

\section{Sous un ciel couleur cayenne (2017)}

+ [S. n.], «Des recueils simples et percutants qui jouent avec la langue», Radio-Canada, 14 juin 2018, en ligne: https://ici.radio-canada.ca/nouvelle/1105652/livres-ete-suggestionspoesie (page consultée le 25 février 2019).

+ CÔTÉ DUPUIS, Alice, «Sous un ciel couleur cayenne de Patrice Desbiens : savoir voir au-delà», Regroupement des éditeurs canadiens-français, 22 novembre 2017, en ligne: http://refc.ca/42sous-un-ciel-couleur-cayenne-de-patrice-desbiens/ (page consultée le 25 février 2019).

+ PARAYRE, Catherine, "Sous un ciel couleur cayenne», Voix plurielles, vol. XV, nº 1, 2018, p. 283.

+ TARDIF, Dominic, "Vitrine du livre: Sous un ciel couleur cayenne, Patrice Desbiens», Le Devoir, 23 décembre 2017, p. D30.

\section{G. Entretiens avec $l^{\prime}$ auteur}

+ ACQUELIN, José et Yves BOISVERT, «Patrice Desbiens. Un couteau à beurre en plastique volé à l'Académie», Lettres québécoises, n 119, automne 2005, p. 7-10.

+ BÉLANGER, Georges, "Portrait d'auteur: Patrice Desbiens», Francophonies d'Amérique, n 2, 1992, p. 93-100.

+ CHAMBERLAND, François-Xavier, «Patrice Desbiens (26 octobre 1995)», François-Xavier Chamberland, L'Ontario se raconte de A à X. Entrevues radiophoniques, Toronto, Éditions du GREF, coll. «Dont actes», 1999, p. 461-470.

+ DESPATIE, Stéphane, "Patrice Desbiens. Table d'harmonies», Voir, 23 septembre 2004, p. 19.

+ DICKSON, Robert, "Of books and men: Ti-Jean, Patrice, Robert et les autres», Liaison, n ${ }^{\circ} 44$, automne 1987, p. 5-6.

+ GAGNON, Paulette, «Patrice Desbiens: tout a commencé ici», Liaison, n 84, novembre 1995, p. 14-16.

+ LABELLE, Marc, «Entretiens avec Patrice Desbiens», Rauque, n 7, 1987, p. 49-60.

+ TARDIF, Dominic, «Patrice Desbiens: vrai comme chu là», Voir, 13 octobre 2011, en ligne: https://voir.ca/livres/2011/10/13/patrice-desbiens-vrai-comme-chu-la-2/ (page consultée le 25 février 2019).

+ TRUAX, Denise, «Des poètes se prononcent», Liaison, n 13, hiver 1980, p. 21. 